\title{
ON UNIQUENESS IN LINEAR VISCOELASTICITY: A FAMILY OF COUNTEREXAMPLES*
}

\author{
BY \\ A. MORRO (University of Genova, Italy) \\ AND \\ M. FABRIZIO (University of Bologna, Italy)
}

\begin{abstract}
Within the framework of linear viscoelasticity a two-parameter family of relaxation functions are exhibited which allow nonuniqueness of the infinitesimal strain, for a given stress, in the space of sinusoidal-in-time strain histories. These relaxation functions are shown to be compatible with thermodynamics and to have a positive equilibrium elastic modulus.
\end{abstract}

1. Introduction. In a paper that appeared in 1979 [1], Fichera exhibited an example of a relaxation function, for linear viscoelastic bodies, which, while being compatible with the principle of fading memory, does not allow the solution to the quasi-static problem to exist or to be unique. This nonexistence or nonuniqueness result gave rise to a renewed interest into the subject of viscoelastic relaxation functions (see, e.g., [2-7]). On the one hand some research has been undertaken about thermodynamic requirements in order to distinguish between physical and unphysical relaxation functions and hence to recognize the possible unphysical origin of anomalous behaviours. On the other, it was clarified by Fichera [7] that even relaxation functions compatible with thermodynamics may lead to nonexistence or nonuniqueness if suitably large function spaces for the strain histories are adopted. So a strict connection has been pointed out between the set of restrictions on the relaxation function and the function space ensuring existence and uniqueness. This connection in turn indicates that a precise characterization of relaxation functions is in order.

It is the aim of this note to show that there exists a relaxation function, indeed a family of relaxation functions, which, while complying with the requirements of thermodynamics, does not allow the quasi-static problem of linear viscoelasticity to have a unique solution in the space of sinusoidal-in-time strain histories. Besides being interesting for the

\footnotetext{
*Received April 17, 1986. The research leading to this work was partially supported by GNFM-CNR and the Italian Ministry of Education through the 40\% Project "Problemi di evoluzione nei fluidi e nei solidi".
} 
structure of the relaxation function, the example so elaborated opens new questions within the thermomechanics of continuous media. In particular, since we are accustomed to the use of sinusoidal histories for continuous media, we are led to suspect that the present status of thermomechanics suffers from some deficiency foremost in conjunction with the second law. For instance, a more restrictive statement of the second law makes the present counterexample inapplicable.

Although the following analysis applies equally well to three-dimensional bodies, for the sake of formal simplicity we restrict our attention to one-dimensional bodies.

2. A family of relaxation functions compatible with thermodynamics. By definition, an isotropic viscoelastic solid when subject to a simple shear state of deformation will have a corresponding stress which remains nonzero as long as the state of deformation is kept constant (cf. [8, 9]). Letting $G(s), s \in[0, \infty)$, be the one-dimensional stress-strain relaxation function, this implies that the equilibrium elastic modulus

$$
G(\infty)=\lim _{s \rightarrow \infty} G(s)
$$

satisfies the inequality

$$
G(\infty)>0
$$

Incidentally, the property $G(\infty)=0$ characterizes viscoelastic fluids.

Let $\hat{G}_{s}^{\prime}$ be the half-range sine transform of the derivative $G^{\prime}$ of $G$, namely

$$
\hat{G}_{s}^{\prime}(\omega)=\int_{0}^{\infty} G^{\prime}(s) \sin (\omega s) d s .
$$

It has long been known (cf. $[10,11])$ that, as a consequence of thermodynamics, $\hat{G}_{s}^{\prime}$ must satisfy the inequality

$$
\hat{G}_{s}^{\prime}(\omega) \leqslant 0, \quad \omega>0 .
$$

It is a remarkable result, proved in [6], that (2) is also sufficient for the validity of the second law in the form of the Clausius property [12]. Moreover, it follows from (2) that [6]

$$
G(0)-G(s) \geqslant 0, \quad s \in[0, \infty),
$$

whence we get the conditions $[13,14]$

$$
\begin{gathered}
G(0)-G(\infty) \geqslant 0, \\
G^{\prime}(0) \leqslant 0 .
\end{gathered}
$$

Consider the two-parameter family of relaxation functions

$$
G(s)=\frac{\alpha(\alpha-2)}{16 \beta^{3}}-\int_{0}^{s}\left(r^{2}-\frac{\alpha-1}{\beta} r+\frac{\alpha^{2}}{8 \beta^{2}}\right) \exp (-\beta r) d r,
$$

with $\alpha \in(6,8), \beta>0$; of course

$$
G(0)=\frac{\alpha(\alpha-2)}{16 \beta^{3}}, \quad G^{\prime}(s)=-\left(s^{2}-\frac{\alpha-1}{\beta} s+\frac{\alpha^{2}}{8 \beta^{2}}\right) \exp (-\beta s) .
$$


Straightforward calculations show that the functions (3) satisfy the conditions (1), (2). To see this we observe first that

$$
G(\infty)=G(0)+\int_{0}^{\infty} G^{\prime}(r) d r=-\frac{(\alpha-6)(\alpha-8)}{16 \beta^{3}} ;
$$

because $\alpha \in(6,8)$ the validity of (1) is apparent. Next we evaluate $\hat{G}_{s}^{\prime}$ to get

$$
\hat{G}_{s}^{\prime}(\omega)=-\frac{\omega \alpha^{2}}{8 \beta^{2}\left(\beta^{2}+\omega^{2}\right)^{3}}\left(\omega^{2}-\frac{8-\alpha}{\alpha} \beta^{2}\right)^{2} .
$$

Hence it follows that (2) holds. Indeed, letting

$$
\bar{\omega}=\sqrt{\frac{8-\alpha}{\alpha}} \beta
$$

we have

$$
\begin{aligned}
& \hat{G}_{s}^{\prime}(\omega)=0 \text { for } \quad \omega=0, \bar{\omega}, \\
& \hat{G}_{s}^{\prime}(\omega)<0 \text { for } \quad \omega \neq 0, \bar{\omega} .
\end{aligned}
$$

As an aside we notice that the functions (3) are not monotone in that $G^{\prime}(s)>0$ as $s$ runs between the (positive) roots of $G^{\prime}(s)=0$. However, this does not prevent (3) from being compatible with thermodynamics.

For later convenience we observe that a direct integration yields

$$
\int_{0}^{\infty} G^{\prime}(s) \cos (\bar{\omega} s) d s=-\frac{\alpha(\alpha-2)}{16 \beta^{3}} .
$$

Accordingly, in addition to satisfying (1) and (2), the functions (3) meet the condition

$$
G(0)+\int_{0}^{\infty} G^{\prime}(s) \cos (\bar{\omega} s) d s=0 .
$$

3. Nonuniqueness in linear viscoelasticity. Consider a linear (one-dimensional) viscoelastic body $\mathscr{B}$. Let $t \in R$ denote the time and $x \in R$ the position of the particle under consideration of $\mathscr{B}$. The stress-strain constitutive relation may be written as

$$
T(x, t)=G(0) E(x, t)+\int_{0}^{t} G^{\prime}(s) E(x, t-s) d s .
$$

In the quasi-static approximation the equation of motion reduces to

$$
\frac{d T}{d x}+b=0
$$

where $b$ is the body force (per unit length). Then, if $E(x, t)$ is differentiable with respect to $x$, whenever the solution $E(x, t)$ to $(6)$ for a fixed stress $T(x, t)$ is not unique then the solution $E(x, t)$ to (7) as well is not unique. Examine now the possibility of nonunique solutions $E(x, t)$ to (6). For simplicity take $b(x, t)=0$. Evidently $E(x, t)=0$ is a solution. Let

$$
E(x, t)=E_{0}(x) \exp (i \bar{\omega} t)
$$


with $E_{0}(x)$ differentiable but otherwise arbitrary. Then (6) with $T=0$ yields

$$
G(0) \exp (i \bar{\omega} t)+\int_{0}^{\infty} G^{\prime}(s) \exp [i \bar{\omega}(t-s)] d s=0
$$

Thus nonuniqueness holds provided that

$$
\begin{gathered}
G(0)+\int_{0}^{\infty} G^{\prime}(s) \cos (\bar{\omega} s) d s=0, \\
\int_{0}^{\infty} G^{\prime}(s) \sin (\bar{\omega} s) d s=0 .
\end{gathered}
$$

The relaxation functions (3) meet these conditions because of (4), (5) and hence they allow nonuniqueness of the solution to the quasi-static problem of viscoelasticity.

4. Conclusions. The crucial point in proving the nonuniqueness property of the solution $E(x, t)$ to $(6)$ is the existence of a circular frequency $\omega>0$ such that $\hat{G}_{s}^{\prime}(\omega)=0$. On the other hand, the vanishing of $\hat{G}_{s}^{\prime}$ is allowed by thermodynamics merely because the second law (Clausius property) for cyclic processes requires the entropy production to be nonnegative, rather than strictly positive. This observation suggests that nonuniqueness could ultimately be induced by the expression of the second law.

The statement of the second law in standard textbooks of thermodynamics specifies that the equality sign refers to reversible processes and the inequality sign to irreversible processes (cf. $[15,16])$. Now, roughly speaking, the process associated with a sinusoidal time evolution of the strain in a viscoelastic solid is likely to be irreversible and then we should have the inequaltiy (2) replaced with

$$
\hat{G}_{s}^{\prime}(\omega)<0, \quad \omega>0 .
$$

This condition would rule out the present counterexamples to uniqueness in viscoelasticity.

Based on these observations we believe that a more precise statement of the second law is in order. Any conceptual progress, however, seems to be related to the definition of irreversible process (cf. [17]). The distinction between reversible and irreversible processes will be the subject of a future paper.

\section{REFERENCES}

[1] G. Fichera, Avere una memoria tenace crea gravi problemi, Arch. Rat. Mech. Anal. 70, 101-112 (1979)

[2] D. Graffi, On the fading memory, Applicable Analysis 15, 295-311 (1983)

[3] G. Capriz and M. E. Gurtin, letter to G. Fichera referred to in [4]

[4] G. Fichera, Sul principio della memoria evanescente, Rend. Sem. Mat. Univ. Padova 68, 245-259 (1982)

[5] M. Fabrizio and A. Morro, Thermodynamic restrictions on the relaxation functions in linear viscoelasticity, Mech. Res. Comm. 12, 101-105 (1985)

[6] M. Fabrizio and A. Morro, Viscoelastic relaxation functions compatible with thermodynamics, J. Elasticity, to appear

[7] G. Fichera, On linear viscoelasticity, Mech. Res. Comm. 12, 241-242 (1985)

[8] R. M. Christensen, Theory of viscoelasticity, Academic Press, 1971

[9] A. C. Pipkin, Lectures on viscoelasticity theory, Springer, 1972

[10] D. Graffi, Sui problemi della ereditarietà lineare, Nuovo Cimento 5, 53-71 (1928) 
[11] M. J. Leitman and G. M. C. Fisher, The linear theory of viscoelasticity, Encyclopedia of physics VIa/3. Springer, 1973

[12] B. D. Coleman and D. R. Owen, A mathematical foundation for thermodynamics, Arch. Rat. Mech. Anal. 54, 1-104 (1974)

[13] B. D. Coleman, On thermodynamics, strain impulses and viscoelasticity, Arch. Rat. Mech. Anal. 17, 230-254 (1964)

[14] R. M. Bowen and P. J. Chen, Thermodynamic restriction on the initial slope of the stress-relaxation function, Arch. Rat. Mech. Anal. 51, 278-284 (1973)

[15] E. Fermi, Thermodynamics, Dover, 1956; §11

[16] M. W. Zemanski, Heat and thermodynamics, McGraw-Hill, 1957; \$10.9

[17] M. Fabrizio, An existence and uniqueness theorem in quasi-static viscoelasticity, to appear 